\title{
Control of oocyte meiotic maturation and fertilization*
}

\author{
David Greenstein ${ }^{\mathcal{S}}$, Department of Cell and Developmental Biology, \\ Vanderbilt University School of Medicine, Nashville, TN 37232 USA
}

\section{Table of Contents}

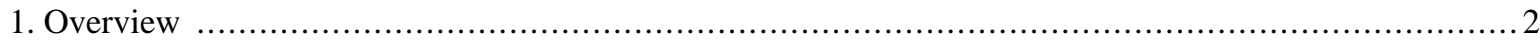

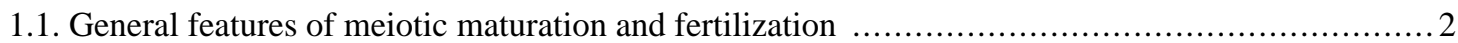

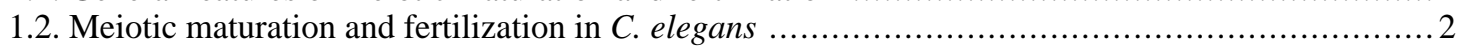

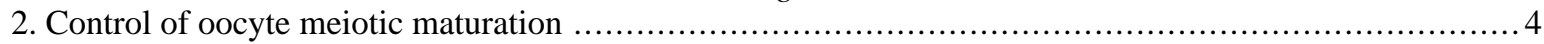

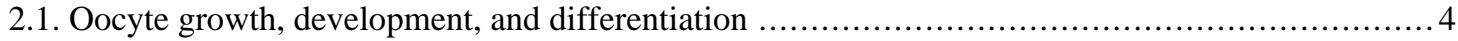

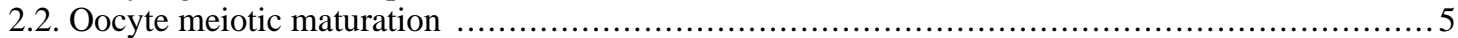

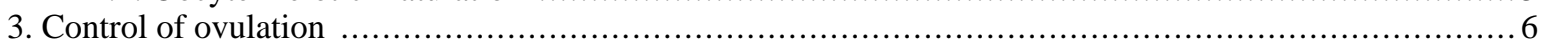

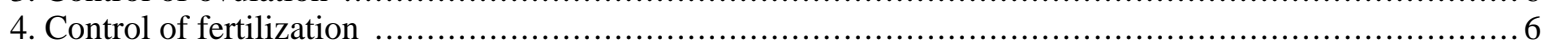

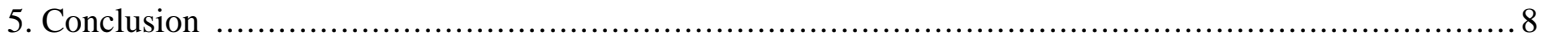

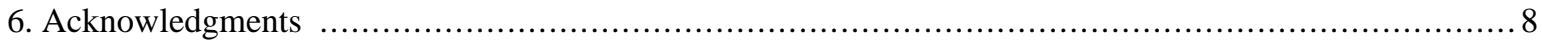

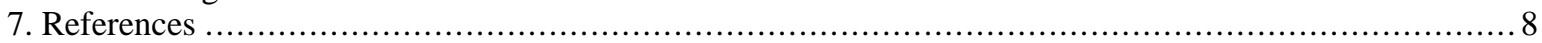

\begin{abstract}
Sexual reproduction depends upon meiosis for the generation of haploid gamete nuclei, which unite after fertilization to form the diploid zygote. The oocytes of most animal species arrest during meiotic prophase, and complete meiosis in response to intercellular signaling in a process called meiotic maturation. Oocyte meiotic maturation is defined by the transition between diakinesis and metaphase of meiosis $I$ and is accompanied by nuclear envelope breakdown, rearrangement of the cortical cytoskeleton, and meiotic spindle assembly. Thus, the meiotic maturation process is essential for meiosis and prepares the oocyte for fertilization. In $C$. elegans, the processes of meiotic maturation, ovulation, and fertilization are temporally coupled: sperm utilize the major sperm protein as a hormone to trigger oocyte meiotic maturation, and in turn, the maturing oocyte signals its own ovulation thereby facilitating fertilization. This chapter highlights recent advances in understanding meiotic maturation signaling and gametic interactions required for fertilization.
\end{abstract}

\footnotetext{
*Edited by Judith Kimble and Susan Strome. Last revised March 24, 2005. Published December 28, 2005. This chapter should be cited as: Greenstein, D. Control of oocyte meiotic maturation and fertilization (December 28, 2005), WormBook, ed. The C. elegans Research Community, WormBook, doi/10.1895/wormbook.1.53.1, http://www.wormbook.org.

Copyright: (C) 2005 David Greenstein. This is an open-access article distributed under the terms of the Creative Commons Attribution License, which permits unrestricted use, distribution, and reproduction in any medium, provided the original author and source are credited.

${ }_{\text {§ }}$ To whom correspondence should be addressed. E-mail: david.greenstein@vanderbilt.edu
} 


\section{Overview}

\subsection{General features of meiotic maturation and fertilization}

Sexual reproduction depends upon meiosis for the generation of haploid gamete nuclei, which unite after fertilization to form the diploid zygote. Despite this universal requirement, meiosis is regulated differently in oocytes and spermatocytes. Whereas spermatocytes proceed through the meiotic divisions uninterrupted, oocytes almost invariably arrest during one, and sometimes two stages following premeiotic DNA replication and meiotic recombination, depending on the species. This unique characteristic of oocyte meiosis, as well as its close temporal association with fertilization, was recognized early by developmental biologists (see Wilson, 1925), who coined the term meiotic maturation for the suite of physiological changes occurring in oocytes just before zygote formation. Oocyte meiotic maturation is defined by the transition between diakinesis and metaphase of meiosis I and is accompanied by nuclear envelope breakdown, rearrangement of the cortical cytoskeleton, and meiotic spindle assembly (Figure 1). Thus, the meiotic maturation process is essential for meiosis and may prepare the oocyte for fertilization.

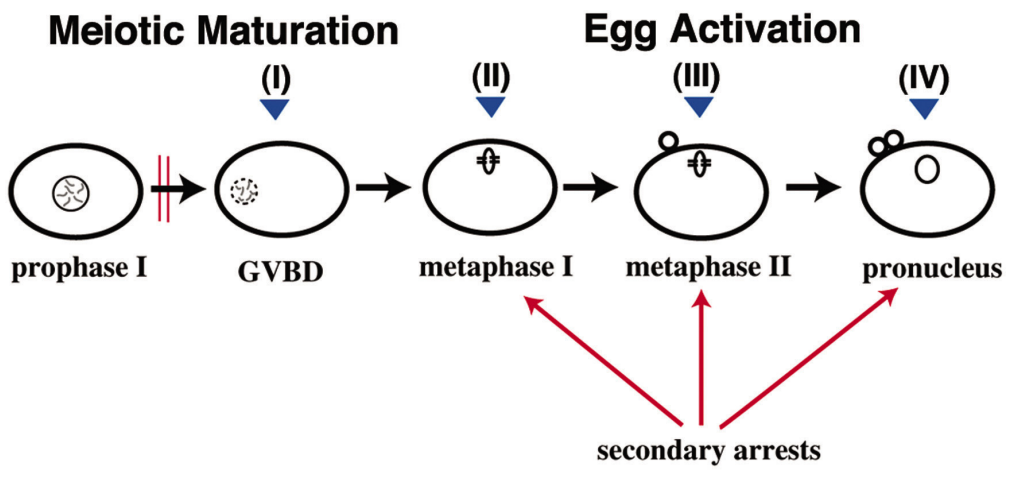

\footnotetext{
= point of fertilization (depends on species)

= primary arrest (often released by hormonal stimulus)
}

Figure 1. Oocyte Meiotic Maturation and Egg Activation. The oocytes of most animal species arrest in meiotic prophase I (reviewed by Masui and Clarke, 1979; Masui, 2001). In response to a hormonal stimulus (1-methyladenine in starfish; progesterone in Xenopus; MSP in C. elegans), oocytes begin meiotic maturation: the nuclear envelope breaks down (GVBD), as the oocyte enters M-phase from prophase. The point of fertilization is species-specific. In the case of $C$. elegans (I) fertilization occurs after maturation but prior to completion of meiosis I. In $C$. elegans, fertilization is required for completing both meiotic divisions. For most insects (II), fertilization occurs at metaphase or anaphase I. In most vertebrates (III), fertilization occurs at metaphase II. Sea urchins complete meiosis before fertilization (IV).

To ensure successful fertilization, oocyte meiotic maturation must be coordinated with other cellular events during oogenesis, including growth, meiotic chromosome reorganization, and ovulation. To achieve this coordination, intercellular signals regulate oocyte meiotic progression (reviewed by Ferrell, 1999; Masui, 2001). The timing of the meiotic divisions with respect to fertilization varies among species, likely reflecting the diversity of reproductive strategies observed in nature (Figure 1). Despite these differences in timing, extensive studies reveal striking conservation in the molecular underpinnings of oocyte meiotic maturation among different animals. It was classic studies of oocyte meiotic maturation in amphibian oocytes that led to the discovery of the Maturation Promoting Factor (MPF; Masui and Markert, 1971; reviewed by Masui, 2001). Genetic and biochemical analysis of the cell cycle, together with MPF purification, subsequently demonstrated that cyclin-dependent protein kinases are universal regulators of mitotic and meiotic cell cycle progression in eukaryotes (reviewed by Morgan, 1995).

\subsection{Meiotic maturation and fertilization in C. elegans}

C. elegans is an important system for addressing the fundamental events of oocyte meiotic maturation and fertilization, complementing studies in vertebrate systems (reviewed by Hubbard and Greenstein, 2000). Oocytes grow and develop in the proximal gonad arm of adult hermaphrodites (Figure 2A). Apoptotic cell death of germ cells is a conserved feature of oogenesis, occurring in adults near the gonad loop region, where the gonad tube bends ventrally. As germline nuclei transit through the loop region, they exit pachytene, become more fully enclosed by plasma membrane, and form a queue in the proximal gonad arm. The meiotic chromosomes condense as the 
developing oocytes enter diplotene. In diakinesis, six discrete highly condensed bivalents, corresponding to the homologous chromosome pairs, are readily visualized (Figure 2B). Oocytes develop in close association with proximal gonadal sheath cells (Figure 2), smooth muscle-like cells that regulate meiotic maturation and contract to drive ovulation (McCarter et al., 1997; Hall et al., 1999; Miller et al., 2003). Meiotic maturation takes place in an assembly line fashion, such that the most proximal oocyte (often referred to as the -1 oocyte) matures, enters the spermatheca at ovulation, and is fertilized (Figure 2; Movie 1). In adult hermaphrodites, the cycle repeats every $\sim 23$ minutes (McCarter et al., 1999).
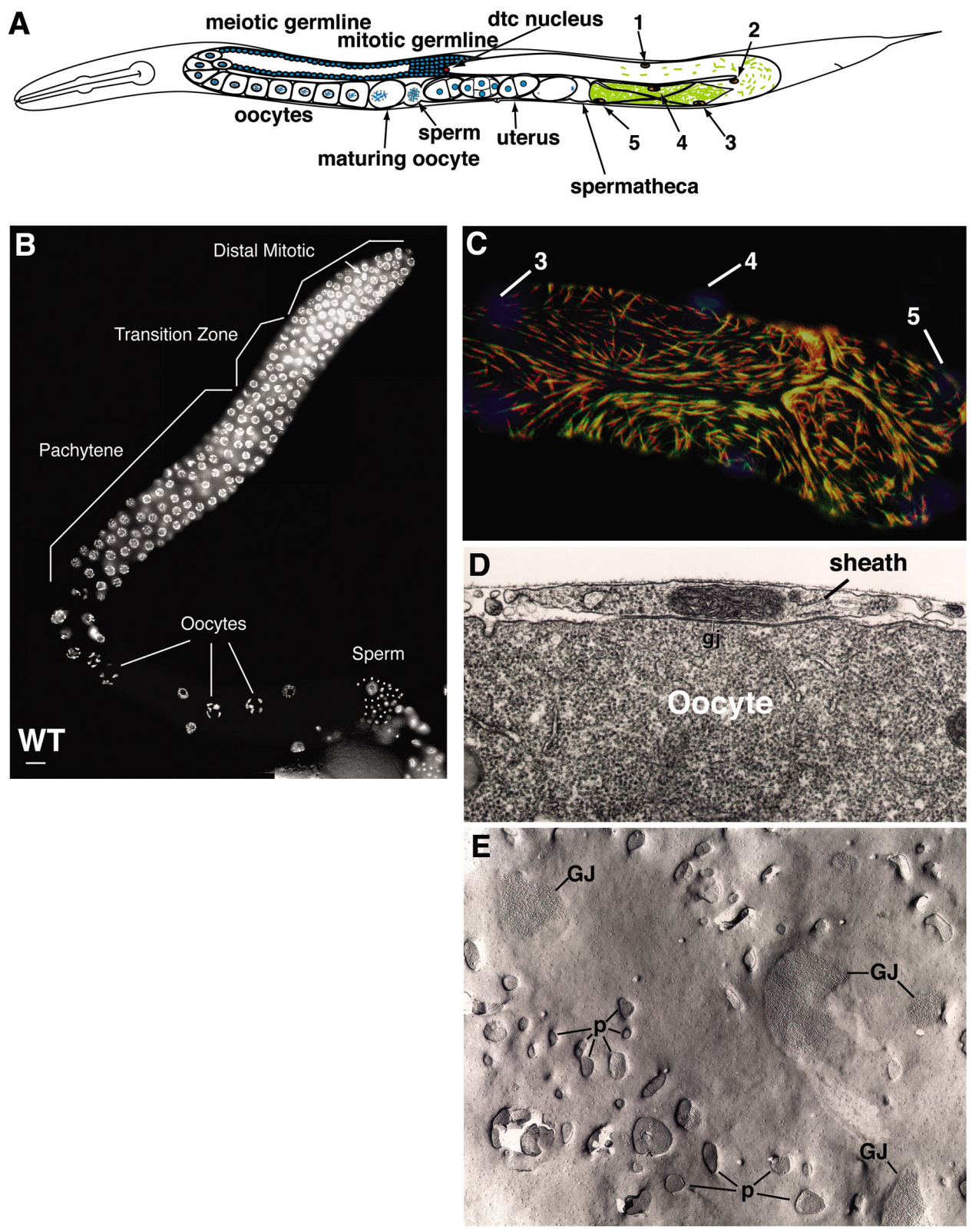

Figure 2. Anatomy Relating to the Control of Meiotic Maturation. (A) Representation of the adult hermaphrodite gonad. The anterior arm (on the left) shows the germ line and the posterior arm (on the right) shows the somatic cells of the gonad arm. Ten gonadal sheath cells cover the gonad arm. The position of one member of each pair is shown (1-5). The proximal sheath cells (pairs 3-5) contract to drive ovulation. (B) Dissected gonad from a wild-type young adult hermaphrodite illustrating oogenic meiotic progression. Bars, $10 \mu \mathrm{m}$. Photograph modified from Berry et al. (1997) with permission. (C) Immunofluorescent micrograph of the contractile proximal gonadal sheath cells (pairs 3-5). Nuclei detected by DAPI staining (blue) and myofilaments in red (MHCA) and green (MHCB). Reproduced from Hubbard and Greenstein (2000) with permission. (D) Gap junction (gj) between oocyte and proximal sheath cell. Photograph modified from Hall et al. (1999) with permission. (E) High magnification view of a freeze-fracture replica of sheath cell 4 covering the -2 oocyte. Clustered intra-membrane particles and pits correspond to gap junctional channels (GJ) between the oocyte and sheath cell. Irregular sheath pores (p) form fenestrae through which the oocyte surface is visible. Photograph modified from Hall et al. (1999) with permission. 


\section{Wild Type Ovulation}

Movie 1. Time-lapse Video of Oocyte Meiotic Maturation and Ovulation. The first ovulation in a young-adult hermaphrodite is shown. Events occur twelve times faster than real time.

In many animals, including many species of sponges, annelids, mollusks, and nematodes, sperm promote the resumption of meiosis in arrested oocytes (Masui, 1985; McCarter et al., 1999). In C. elegans, sperm utilize the major sperm protein (MSP) as a hormone to promote oocyte meiotic maturation and gonadal sheath cell contraction at a distance (Miller et al., 2001). MSP is also the key cytoskeletal element required for amoeboid locomotion of nematode spermatozoa (Italiano et al., 1996). MSP promotes oocyte meiotic maturation, in part by binding the VAB-1 Eph receptor protein-tyrosine kinase on oocytes, and in part by antagonizing an inhibitory somatic gonadal sheath cell pathway (Miller et al., 2003). Since hermaphrodites produce only a fixed number of sperm, meiotic maturation rates are initially high for the first two days of adulthood, but decline as sperm are used for fertilization. In gonochoristic species (e.g., C. remanei), oocytes arrest at diakinesis until the female is inseminated during mating. Likewise, in sex-determination mutants of $C$. elegans, which fully feminize the hermaphrodite gonad, oocytes also arrest until sperm are supplied. Thus, the MSP hormone functions as the linchpin of a sperm-sensing mechanism linking meiotic maturation and sperm availability, thereby ensuring fertilization.

\section{Control of oocyte meiotic maturation}

\subsection{Oocyte growth, development, and differentiation}

\subsubsection{Pachytene progression and oogenesis}

Progression through pachytene to diplotene requires the mitogen-activated protein kinase (MAPK) signaling pathway in the germ line (Church et al., 1995). MAPK activation is also involved in meiotic maturation (see below). Mutations in multiple RAS/MAPK pathway components cause a similar phenotype in which germline nuclei arrest in pachytene. The arrested germline nuclei are displaced from the surface of the gonadal tube and clump before the bend (Church et al., 1995; Hsu et al., 2002).

Whereas the RAS/MAPK pathway is required for fertility in both sexes, the daz-1 gene, a homolog of the human Deleted in Azoospermia gene, is specifically required for progression through pachytene during oogenesis (Karashima et al., 2000). Since daz-1 encodes a ribonucleoprotein-type RNA-binding protein, it may regulate translation during female meiotic progression. $s k r-1$ and $s k r-2$, which encode two of the 21 Skp1-related genes in $C$. elegans, are also required for progression through pachytene during oogenesis (Nayak et al., 2002). SKP-1 and SKP-2 interact with the CUL-1 cullin and are likely to regulate ubiquitin-mediated protein degradation to promote progression through pachytene. The maxi-KH/STAR domain RNA-binding protein GLD-1 is also required for oocyte differentiation (Francis et al., 1995; Jones and Schedl, 1995). In the absence of gld-1, germ cells destined to become oocytes enter meiosis, proceed to pachytene, but then exit meiosis, reentering the mitotic cell cycle to form a germline tumor (Francis et al., 1995). GLD-1 functions as a translational repressor protein (Jan et al., 1999; Lee and Schedl, 2001) that also protects its mRNA targets from nonsense-mediated decay (Lee and Schedl, 2004). GLD-1 protein levels are highest during pachytene, drop sharply as germ cells progress to diplotene, and decrease to undetectable levels in growing oocytes (Jones et al., 1996). This observation suggested that gld- 1 may prevent the translation of proteins needed for oocyte growth and development in early meiotic prophase. The isolation of GLD-1 mRNA targets by immunoprecipitation identified candidate genes involved in oocyte development or function (Lee and Schedl, 2001).

\subsubsection{Oocyte growth}

Oocytes grow in the proximal gonad arm, and accumulate yolk lipoprotein particles. The vitellogenins, protein components of yolk particles, are synthesized in the intestine (Kimble and Sharrock, 1983) and secreted into the 
pseudocoelom. Yolk lipoprotein particles appear to pass through the gonadal basal lamina, move through pores in the gonadal sheath cells (Figure 2E), are endocytosed, and stored in membrane-bounded granules within oocytes (Hall et al., 1999). Endocytosis, of yolk lipoprotein particles requires the RME-2 yolk receptor, a member of the low-density lipoprotein receptor superfamily (Grant and Hirsh, 1999). The spatial pattern of rme-2 expression is controlled by gld-1 (Lee and Schedl, 2001) and atx-2 (Ciosk et al., 2004). The vitellogenins are cholesterol-binding proteins and RME-2 receptor-mediated endocytosis is required for normal transport of cholesterol into oocytes (Matyash et al., 2001). Since adult hermaphrodites turn over the entire volume of the gonad every 6.5 hours, (Hirsh et al., 1976), oocyte growth must be highly regulated. Understanding oocyte growth control is a frontier for the field. Signaling pathways that intersect with meiotic maturation and MAPK pathways appear to be involved (Gutch et al., 1998; T. Schedl, unpublished results).

\subsection{Oocyte meiotic maturation}

\subsubsection{Description of anatomy and timing of events}

Oocyte meiotic maturation can be viewed by time-lapse videomicroscopy of living animals (Ward and Carrel, 1979; Movie 1). The timing of landmark events during meiotic maturation (McCarter et al., 1999) and the ultrastructure of the proximal gonad (Hall et al., 1999) have been described. The nuclear envelope of the most proximal oocyte breaks down $\sim 5$ min prior to ovulation as it enters meiotic M-phase from prophase (McCarter et al., 1999). During maturation, the oocyte also undergoes a structural change termed cortical rearrangement (McCarter et al., 1999). These changes within the oocyte coincide with a reproducible sequence of somatic motor events mediated by the contractile proximal sheath cells (Figure 2A, Figure 2D) and the distal spermatheca resulting in ovulation. The gonadal sheath cells form gap junctions with oocytes (Figure 2D, Figure 2E) and regulate meiotic maturation and ovulation (Greenstein et al., 1994; McCarter et al., 1997; Rose et al., 1997; Miller et al., 2003).

\subsubsection{The MSP signal}

In a landmark study, McCarter et al., (1999) showed that a sperm-associated signal promotes oocyte meiotic maturation independent of fertilization. In females (genetically altered XX animals that produce no sperm), oocytes mature and are ovulated at low rates $(<0.1$ maturations per gonad per hr). Mating to wild-type males, or fertilization incompetent sperm defective (spe) mutants restores the normal rate of oocyte maturation when sperm are plentiful ( 2.5 maturations per gonad per hr). Until recently, a key unanswered question in the field concerned the nature of the sperm signal for oocyte meiotic maturation. Miller et al. (2001) demonstrated that the major sperm protein (MSP), the central cytoskeletal element required for the actin-independent motility of nematode spermatozoa, has a dual role in C. elegans reproduction, functioning as a hormone for oocyte meiotic maturation and gonadal sheath cell contraction. An in vivo bioassay was developed in which sperm-conditioned medium or sperm lysates were injected into the uterus of unmated fog-2 female animals, and oocyte meiotic maturation and gonadal sheath cell contraction were monitored by time-lapse videomicroscopy. The bioactive factors were purified to homogeneity with reversed-phase high-performance liquid chromatography and shown to contain only MSP by mass spectrometry. Injection of MSP antibodies into the uterus of hermaphrodites results in a reduction in ovulation rates, consistent with the hypothesis that MSP is an endogenous signal. C. elegans MSP can signal in C. remanei (Miller et al., 2001) and Ascaris MSP can signal in C. elegans (M. Kosinski and D.G., unpublished results), consistent with observations of heterospecific matings in the genus Caenorhabditis (Hill and L'Hernault, 2001).

MAPK activation plays critical roles in regulating meiotic progression (Ferrell, 1999). In C. elegans, the MAPK pathway is required for pachytene progression (Church et al., 1995) and is important for meiotic maturation (M.-H. Lee and T. Schedl, pers. comm.). Proximal oocytes exhibit MAPK activation in the presence of sperm (Miller et al., 2001; Page et al., 2001), and MSP was shown to be sufficient to activate MAPK in oocytes (Miller et al., 2001). MSP begins as a cytoplasmic protein and does not possess a signal sequence. Recent results suggest that MSP is exported from spermatids and spermatozoa by a vesicle budding mechanism (M. Kosinski, K. McDonald, J. Schwartz, I. Yamamoto, and D. G., unpublished results).

\subsubsection{MSP signaling and the sperm-sensing mechanism}

The identification of MSP's signaling role posed the problem of how oocytes and sheath cells sense MSP. A key advance was the identification of the VAB-1 Eph receptor protein-tyrosine kinase as an MSP receptor (Miller et al., 2003; reviewed by Kuwabara, 2003). Recent results show that the VAB-1 ectodomain directly binds MSP in vitro (H. Cheng and D. G., unpublished results). The observation that $v a b-1$ null mutant oocytes respond to and bind MSP, suggests the involvement of additional MSP receptors. vab-l was shown to function in parallel to a somatic 
gonadal pathway (Miller et al., 2003), defined by the POU-class homeobox gene ceh-18 (Greenstein et al., 1994; Rose et al., 1997). These parallel pathways negatively regulate oocyte maturation and MAPK activation in hermaphrodite and female gonads. Eliminating vab-1 and ceh-18 function removes the dependence of meiotic maturation and ovulation on the presence of sperm. Therefore, this meiotic control mechanism resembles a cell cycle checkpoint (Hartwell and Weinert, 1989) and may confer a selective advantage in hermaphrodite and female species by conserving metabolically costly oocytes when sperm are unavailable for fertilization.

Positive effectors of oocyte meiotic maturation have been identified in C. elegans, including the cyclin-dependent kinase homolog CDK-1 (Boxem et al. 1999) and the polo-like kinase homolog PLK-1 (Chase et al. 2000). The zinc finger domain-containing proteins OMA-1 and OMA-2 are redundantly required for oocyte maturation and ovulation (Detwiler et al. 2001). In oma-1; oma-2 double mutants, MAPK activation is not sustained and nuclear envelope breakdown does not occur. OMA-1 and OMA-2 may function upstream of two conserved cell cycle regulators, the MYT1-related kinase WEE-1.3 and CDK-1 (Detwiler et al. 2001). Since OMA-1 and OMA-2 contain zinc finger domains, they could function as regulators of translation or as regulators of proteolysis (DeRenzo et al., 2003).

\section{Control of ovulation}

The ovulatory process represents an attractive physiological model for how intercellular signaling affects smooth muscle function. Ovulation involves the coordinated function of the gonadal sheath cells, specialized smooth-muscle-like cells that surround oocytes (Figure 2). During ovulation, the proximal gonadal sheath cells contract rapidly, the distal constriction of the spermatheca dilates, and sheath cells pull the distal spermatheca over the mature oocyte (Movie 1). The maturing oocyte signals its own ovulation in two ways: it modulates sheath contractions, which includes an increase in contraction rate and intensity during ovulation, and it induces spermathecal dilation during ovulation (McCarter et al., 1999). Spermathecal dilation is regulated by a LIN-3(EGF)/LET-23(EGF-receptor) signal transduction pathway mediating the signal from oocyte to the distal spermatheca (Clandinin et al., 1998; T. Schedl, unpublished results). The let-23 pathway required for spermathecal dilation during ovulation is let-60(ras)-independent and involves a downstream IP3-mediated pathway (Clandinin et al., 1998). Dominant gain-of-function mutations in let-60(ras) also perturb ovulation, suggesting that ras-dependent pathways are also involved (T. Schedl, unpublished results). Mutations in two genes, lfe-l/itr-l and lfe-2, (gain-of-function and loss-of-function, respectively) were isolated in a genetic screen for suppressors of let-23 sterility defects. Ife-1/itr- 1 and lfe-2 genes encode an inositol $(1,4,5)$ triphosphate receptor and an inositol $(1,4,5)$ triphosphate-3-kinase, respectively (Clandinin et al., 1998). These results suggest that spermathecal dilation is likely to be dependent on calcium release regulated by IP3. Consistent with this possibility, a mutant allele of ipp-5, which encodes a type I 5-phosphatase, predicted to lower IP3 levels, exhibits a novel ovulation phenotype in which the spermatheca overextends, thereby ovulating two oocytes per cycle (Bui and Sternberg, 2002). Mutations in plc-1, which encodes a phospholipase $\mathrm{C}$ epsilon homolog, or plc-l(RNAi), also result in spermathecal entry and exit defects (Kariya et al., 2004; Yin et al., 2004). let-23-mediated IP3 signaling also plays a role in promoting the ovulatory gonadal sheath cell contractions (Yin et al., 2004).

Mutations that lead to defective ovulation cause an endomitotic oocyte (Emo) phenotype (Iwasaki et al., 1996). When oocytes are retained in the gonad arm due to defective ovulation, they undergo multiple rounds of nuclear envelope breakdown (M-phase entry) and S-phase, and become highly polyploid. A large class of genes can mutate to an Emo phenotype and these include emo-1 (Iwasaki et al., 1996), mup-2 (Myers et al., 1996), mel-11 (Wissmann et al., 1999), tropomyosin, and troponin C (Ono and Ono, 2004) as well as genes involved in sheath or spermathecal development (Kostic et al., 2003; Aono et al., 2004). In the future, studies of the physiology of ovulation will no doubt benefit from the ability to measure calcium concentrations in the gonad (Samuel et al., 2001) and to record oocyte ion channels (Rutledge et al., 2001).

\section{Control of fertilization}

Successful fertilization depends on a series of gametic interactions in which spermatozoa and oocyte recognize each other, bind, and fuse (reviewed by Hardy, 2002). Egg activation (the process whereby the oocyte completes the meiotic divisions), a block to polyspermy, and activation of the embryonic program are dependent on fertilization. C. elegans provides a promising experimental system for examining fertilization because of the availability of fertilization-defective mutants (L'Hernault et al., 1988; reviewed by Singson, 2001). The central finding for the field was the phenotypic characterization and molecular cloning of spe-9, a gene whose function is required in sperm for fertilization (Singson et al., 1998). spe-9 spermatozoa are fertilization defective, despite being able to crawl, signal 
meiotic maturation, and contact ovulated oocytes. spe-9 mutations do not affect spermiogenesis, and the mutant spermatozoa have a normal morphology at the EM level. spe-9 encodes a transmembrane protein containing an extracellular domain with 10 EGF-like repeats. Based on these results, Singson et al. (1998) proposed that spe-9 may function as a ligand for an oocyte receptor. Analysis of spe-9-deletion derivatives using a transgenic assay is consistent with this proposal and indicates that the EGF-like repeats and the transmembrane segment are required for function (Putiri et al., 2004). By contrast, the cytoplasmic domain was dispensable, ruling out the possibility that this region plays a signaling role within spermatozoa. spe-9 localizes to the membrane of spermatids, but reorganizes to the pseudopod during spermiogenesis (Zannoni et al., 2003; Figure 3). Based on this result, Zannoni et al. (2003) proposed that the spermatozoan interacts with the oocyte cell surface in a "pseudopod first" manner. While there are no published ultrastructural observations of fertilization events in C. elegans, Foor (1968) reported a striking series of EM observations of fertilization in Ascaris lumbricoides, which provided evidence for initial contact of the pseudopod with the mature oocyte, fusion of cell membranes during fertilization, and first entry of the pseudopodal extension. The identification of oocyte cell surface molecules that mediate fertilization interactions is an exciting area for future work.
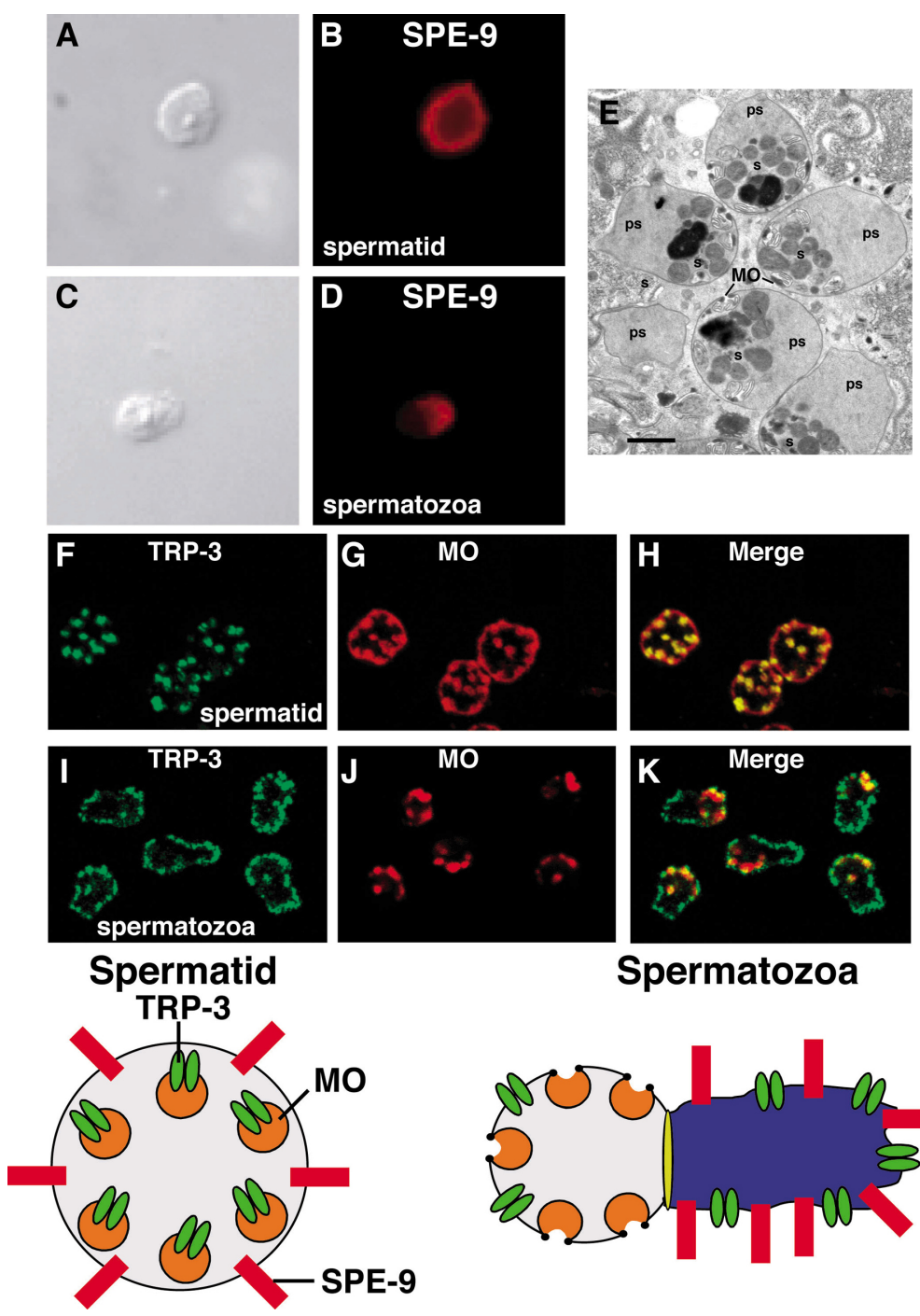

Spermatozoa

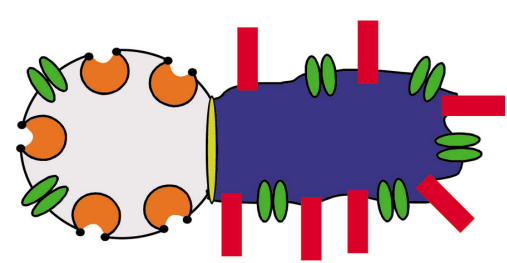

Figure 3. Dynamic Localization of Two Membrane Proteins Required for Fertilization. (A-D) Localization of SPE-9 in spermatids (A and B) and spermatozoa (C and D). SPE-9 localizes to the plasma membrane of spermatids. After spermiogenesis, SPE-9 is enriched at the plasma membrane of the pseudopod. Photographs reproduced from Zannoni et al. (2004), and used with permission. (E) High pressure freezing transmission electron microscopy of spermatozoa (s) in the spermatheca. Membranous organelles (MO) and pseudopods (ps) are indicated. During spermiogenesis (not shown) the MOs fuse with the plasma membrane and maintain a persistant fusion pore, flanked by an electron-dense collar. Electron micrograph courtesy of Kent McDonald. Bar, $1 \mu \mathrm{m}$. 
Further studies of fertilization-defective mutations of the spe-9 class promise essential information on gametic interactions needed for fertilization. These mutations include spe-13, spe-38, spe-42, fer-14, and trp-3 (Singson et al., 1998; Xu and Sternberg, 2003; A. Singson, T. Kroft, and S. L'Hernault, unpublished results). Xu and Sternberg (2003) reported an extensive characterization of trp-3 (aka spe-41), which encodes a member of the transient receptor potential (TRP) superfamily of cation channels. While trp-3 plays a critical role in fertilization, it is not absolutely essential because hermaphrodites homozygous for likely trp-3 null mutations have brood sizes that are approximately 5\% of the wild type. TRP-3 displays a dynamic localization during spermiogenesis. TRP-3 localizes to the MOs in spermatids and translocates to the plasma membrane of the cell body and pseudopod during spermiogenesis (Figure 3). Calcium imaging studies provided evidence that TRP-3 may function as a store-operated calcium entry (SOCE) channel in spermatozoa. In addition to SOCE channels, spermatozoa contain constitutively active calcium-permeable (CAC) channels. While trp-3 mutant spermatozoa exhibit lower SOCE channel activity than the wild type, the CAC channel activity is normal. These results strongly suggest that TRP cation channel activity plays an important role in fertilization. In mammals, TRP channels play roles in the acrosome reaction (Jungnickel et al., 2001) and sperm motility (Castellano et al., 2003). Additional members of this large channel family may have conserved functions in fertilization.

\section{Conclusion}

Meiotic maturation and fertilization are fundamental developmental events, which have been extensively studied for over a hundred years. Yet, the molecular mechanisms underlying these critical processes remain incompletely understood. Many exciting biological questions surrounding these events remain. For instance, it is unclear how meiotic maturation events actually prepare the oocyte for fertilization. The sequence of molecular events needed at fertilization and their interdependencies are uncharacterized. How fertilization results in egg activation and triggers the embryonic program is largely unknown. Recent progress described here gives confidence that $C$. elegans provides a fertile field for answering basic questions about the fundamental developmental mechanisms of oocyte meiotic maturation and fertilization.

\section{Acknowledgments}

I thank Michael Miller, Tim Schedl, Andy Singson, and Steve L'Hernault for communicating unpublished results and for discussions. Thanks to Kent McDonald for high-pressure freezing electron microscopy. Thanks to Michael Miller, Sean Xu, and Andy Singson for assistance with the figures. Judith Kimble, Mary Kosinski, Steve L'Hernault, Tim Schedl, and Susan Strome provided valuable comments on the manuscript. Work in my laboratory is supported by grants from the National Institutes of Health (GM57173 and GM65115).

\section{References}

Achanzar, W.E., and Ward, S. (1997). A nematode gene required for sperm vesicle fusion. J. Cell Sci. 110, 1073-1081. Abstract

Aono, S., Legouis, R., Hoose, W.A., and Kemphues, K.J. (2004). PAR-3 is required for epithelial cell polarity in the distal spermatheca of C. elegans. Development 131, 2865-2874. Abstract Article

Berry, L.W., Westlund, B., and Schedl, T. (1997). Germ-line tumor formation caused by activation of glp-1, a Caenorhabditis elegans member of the Notch family of receptors. Development 124, 925-936. Abstract

Boxem M, Srinivasan DG, and van den Heuvel, S. (1999). The Caenorhabditis elegans gene ncc-1 encodes a $c d c 2$-related kinase required for $\mathrm{M}$ phase in meiotic and mitotic cell divisions, but not for $\mathrm{S}$ phase. Development 126, 2227-2239. Abstract

Bui, Y.K., and Sternberg, P.W. (2002). Caenorhabditis elegans inositol 5-phosphatase homolog negatively regulates inositol 1,4,5-triphosphate signaling in ovulation. Mol. Biol Cell. 13, 1641-1651. Abstract Article

Castellano, L.E., Trevino, C.L., Rodriguez, D., Serrano, C.J., Pacheco, J., Tsutsumi, V., Felix, R., and Darszon, A. (2003). Transient receptor potential (TRPC) channels in human sperm: expression, cellular localization and involvement in the regulation of flagellar motility. FEBS Lett. 541, 69-74. Abstract Article 
Chase, D., Serafinas, C., Ashcroft, N., Kosinski, M., Longo, D., Ferris, D.K., and Golden, A. (2000). The polo-like kinase PLK-1 is required for nuclear envelope breakdown and the completion of meiosis in Caenorhabditis elegans. Genesis 26, 26-41. Abstract Article

Church, D.L., Guan, K.-L., and Lambie, E.J. (1995). Three genes of the MAP kinase cascade, mek-2, mpk-1/sur-1 and let-60 ras, are required for meiotic cell cycle progression in Caenorhabditis elegans. Development 121, 2525-2535. Abstract

Ciosk, R., DePalma, M., and Priess, J.R. (2004). ATX-2, the C. elegans ortholog of ataxin 2, functions in translational regulation in the germ line. Development 131, 4831-4841. Abstract Article

Clandinin, T.R., DeModena, J.A., and Sternberg, P.W. (1998). Inositol triphosphate mediates a RAS-independent response to LET-23 receptor tyrosine kinase activation in C. elegans. Cell 92, 523-533. Abstract Article

DeRenzo, C., Reese, K.J., and Seydoux, G. (2003). Exclusion of germ plasm proteins from somatic lineages by cullin-dependent degradation. Nature 424, 685-689. Abstract Article

Detwiler, M.R., Reuben, M., Li, X., Rogers, E., and Lin, R. (2001). Two zinc finger proteins, OMA-1 and OMA-2, are redundantly required for oocyte maturation in C. elegans. Dev. Cell 1, 187-199. Abstract Article

Ferrell, J.E., Jr. (1999). Xenopus oocyte maturation: new lessons from a good egg. Bioessays 21, 833-842. Abstract Article

Foor, W.E. (1969). Zygote formation in Ascaris lumbricoides (Nematoda). J. Cell Biol. 39, 119-134. Article

Francis, R., Barton, M.K., Kimble, J., and Schedl, T. (1995). gld-1, a tumor suppressor gene required for oocyte development in Caenorhabidits elegans. Genetics 139, 579-606. Abstract

Grant, B., and Hirsh, D. (1999). Receptor-mediated endocytosis in the Caenorhabditis elegans oocyte. Mol. Biol. Cell 10,4311-4326. Abstract

Greenstein, D., Hird, S., Plasterk, R.H.A., Andachi, Y., Kohara, Y., Wang, B., Finney, M., and Ruvkun, G. (1994). Targeted mutations in the Caenorhabditis elegans POU homeo box gene ceh-18 cause defects in oocyte cell cycle arrest, gonad migration, and epidermal differentiation. Genes Dev. 8, 1935-1948. Abstract

Gutch, M.J., Flint, A.J., Keller, J., Tonks, N.K., and Hengartner, M.O. (1998). The Caenorhabditis elegans SH2 domain-containing protein tyrosine phosphatase PTP-2 participates in signal transduction during oogenesis and vulval development. Genes Dev. 12, 571-585. Abstract

Hall, D.H., Winfrey, V.P., Blaeuer, G., Hoffman, L.H., Furuta, T., Rose, K.L., Hobert, O., and Greenstein D. (1999). Ultrastructural features of the adult hermaphrodite gonad of Caenorhabditis elegans: relations between the germ line and soma. Dev. Biol. 212, 101-123. Abstract Article

Hardy, D.M. ed. (2002). Fertilization (San Diego: Academic Press).

Hartwell, L.H. and Weinert, T.A. (1989). Checkpoints: controls that ensure the order of cell cycle events. Science 246, 629-634. Abstract

Hill, K.L., and L'Hernault, S.W. (2001). Analyses of reproductive interactions that occur after heterospecific matings within the genus Caenorhabditis. Dev Biol. 232, 105-114. Abstract Article

Hirsh, D., Oppenheim, D., and Klass, M. (1976). Development of the reproductive system of Caenorhabditis elegans. Dev. Biol. 49, 200-219. Abstract Article

Hsu, V., Zobel, C.L., Lambie, E.J., Schedl, T., and Kornfeld, K. (2002). Caenorhabditis elegans lin-45 raf is essential for larval viability, fertility and the induction of vulval cell fates. Genetics 160, 481-492. Abstract

Hubbard, E.J., and Greenstein, D. (2000). The Caenorhabditis elegans gonad: a test tube for cell and developmental biology. Dev. Dyn. 218, 2-22. Abstract Article 
Italiano, J.E. Jr., Roberts, T.M., Stewart, M., and Fontana, C.A. (1996). Reconstitution in vitro of the motile apparatus from the amoeboid sperm of Ascaris shows that filament assembly and bundling move membranes. Cell 84, 105-114. Abstract Article

Iwasaki, K., McCarter, J., Francis, R., and Schedl, T. (1996). emo-1, a Caenorhabditis elegans Sec61p gamma homologue, is required for oocyte development and ovulation. J. Cell Biol. 134, 699-714. Abstract Article

Jan E., Motzny, C.K., Graves L.E., and Goodwin, E.B. (1999). The STAR protein, GLD-1, is a translational regulator of sexual identity in Caenorhabditis elegans. EMBO J. 18, 258-269. Abstract Article

Jones, A.R., and Schedl, T. (1995). Mutations in gld-1, a female germ cell-specific tumor suppressor gene in Caenorhabditis elegans, affect a conserved domain also found in Src-associated protein Sam68. Genes Dev. 9, 1491-1504. Abstract

Jones, A.R., Francis, R., and Schedl, T. (1996). GLD-1, a cytoplasmic protein essential for oocyte differentiation, shows stage- and sex-specific expression during Caenorhabditis elegans germline development. Dev. Biol. 180, 165-183. Abstract Article

Jungnickel, M.K., Marrero, H., Birnbaumer, L., Lemos, J.R., and Florman, H.M. (2001). Trp2 regulates entry of Ca2+ into mouse sperm triggered by egg ZP3. Nat. Cell Biol. 3, 499-502. Abstract Article

Kamath, R.S., Fraser, A.G., Dong, Y., Poulin, G., Durbin, R., Gotta, M., Kanapin, A., Le Bot, N., Moreno, S., Sohrmann, M., et al. (2003). Systematic functional analysis of the Caenorhabditis elegans genome using RNAi. Nature 421, 231-237. Abstract Article

Karashima, T., Sugimoto, A., and Yamamoto, M. (2000). Caenorhabditis elegans homologue of the human azoospermia factor DAZ is required for oogenesis but not for spermatogenesis. Development 127, 1069-1079. Abstract

Kariya, K., Kim Bui, Y., Gao, X., Sternberg, P.W., and Kataoka, T. (2004). Phospholipase Cepsilon regulates ovulation in Caenorhabditis elegans. Dev. Biol. 274, 201-210. Abstract Article

Kimble, J. and Sharrock, W.J. (1983). Tissue-specific synthesis of yolk proteins in Caenorhabditis elegans. Dev. Biol. 96, 189-196. Abstract Article

Kostic, I., Li, S., and Roy, R. (2003). cki-1 links cell division and cell fate acquisition in the C. elegans somatic gonad. Dev. Biol. 263, 242-252. Abstract Article

Kuwabara, P.E. (2003). The multifaceted C. elegans major sperm protein: an ephrin signaling antagonist in oocyte maturation. Genes Dev. 17, 155-161. Abstract Article

Lee, M.H., and Schedl, T. (2001). Identification of in vivo mRNA targets of GLD-1, a maxi-KH motif containing protein required for $C$. elegans germ cell development. Genes Dev. 15, 2408-2420. Abstract Article

Lee, M.H., and Schedl, T. (2004). Translation repression by GLD-1 protects its mRNA targets from nonsense-mediated mRNA decay in C. elegans. Genes Dev. 18, 1047-1059. Abstract Article

L'Hernault, S.W., Shakes, D.C., and Ward, S. (1988). Developmental genetics of chromosome I spermatogenesis-defective mutants in the nematode Caenorhabditis elegans. Genetics 120, 435-452. Abstract

Masui, Y. (1985). Meiotic arrest in animal oocytes. In: Biology of Fertilization (Florida: Academic Press), pp. 189-219.

Masui, Y. (2001). From oocyte maturation to the in vitro cell cycle: the history of discoveries of maturation-promoting Factor (MPF) and cytostatic factor (CSF). Differentiation 69, 1-17. Abstract Article

Masui, Y., and Clarke, H.J. (1979). Oocyte maturation. Int. Rev. Cytol. 57, 185-282. Abstract

Masui, Y., and Markert, C.L. (1971). Cytoplasmic control of nuclear behavior during meiotic maturation of frog oocytes. J. Exper. Zool. 177, 129-145. Abstract Article 
Matyash, V., Geier, C., Henske, A., Mukherjee, S., Hirsh, D., Thiele, C., Grant, B., Maxfield, F.R., and Kurzchalia, T.V. (2001). Distribution and transport of cholesterol in Caenorhabditis elegans. Mol. Biol. Cell 12, 1725-1736. Abstract

McCarter, J., Bartlett, B., Dang, T., and Schedl, T. (1997). Soma-germ cell interactions in Caenorhabditis elegans: Multiple events in germline development require the somatic sheath and spermathecal lineages. Dev. Biol. 181, 121-143. Abstract Article

McCarter, J., Bartlett, B., Dang, T., and Schedl, T. (1999). On the control of oocyte meiotic maturation and ovulation in Caenorhabditis elegans. Dev. Biol. 205, 111-128. Abstract Article

Miller, M.A., Nguyen, V.Q., Lee, M.H., Kosinski, M., Schedl, T., Caprioli, R.M., and Greenstein, D. (2001). A sperm cytoskeletal protein that signals oocyte meiotic maturation and ovulation. Science 291, 2144-2147. Abstract Article

Miller, M.A., Ruest, P.J., Kosinski, M., Hanks, S.K., and Greenstein, D. (2003). An Eph receptor sperm-sensing control mechanism for oocyte meiotic maturation in Caenorhabditis elegans. Genes Dev. 17, 187-200. Abstract Article

Morgan, D.O. (1995). Principles of CDK regulation. Nature 374, 131-134. Abstract Article

Myers, C.D., Goh, P.-Y., Allen, T. StC., Bucher, E.A., and Bogaert, T. (1996). Developmental genetic analysis of Troponin $\mathrm{T}$ mutations in striated and nonstriated muscle cells of Caenorhabditis elegans. J. Cell Biol. 132, 1061-1077. Abstract Article

Nayak, S., Santiago, F.E., Jin, H., Lin, D., and Schedl, T. (2002). The Caenorhabditis elegans Skp1-related gene family: diverse functions in cell proliferation, morphogenesis, and meiosis. Curr. Biol. 12, 277-287. Abstract Article

Ono, K., and Ono, S. (2004). Tropomyosin and troponin are required for ovarian contraction in the Caenorhabditis elegans reproductive system. Mol. Biol. Cell 15, 2782-2793. Abstract Article

Page, B.D., Guedes, S., Waring, D., and Priess, J.R. (2001). The C. elegans E2F- and DP--related proteins are required for embryonic asymmetry and negatively regulate Ras/MAPK signaling. Mol. Cell 7, 451-460. Abstract Article

Putiri, E., Zannoni, S., Kadandale, P., and Singson, A. (2004). Functional domains and temperature-sensitive mutations in $S P E-9$, an EGF repeat-containing protein required for fertility in Caenorhabditis elegans. Dev. Biol. 272, 448-459. Abstract Article

Rose, K.L., Winfrey, V.P., Hoffman, L.H., Hall, D.H., Furuta, T., and Greenstein, D. (1997). The POU gene ceh-18 promotes gonadal sheath cell differentiation and function required for meiotic maturation and ovulation in Caenorhabditis elegans. Dev. Biol. 192, 59-77. Abstract Article

Rutledge, E., Bianchi, L., Christensen, M., Boehmer, C., Morrison, R., Broslat, A., Beld, A.M., George, A.L., Greenstein, D., and Strange, K. (2001). CLH-3, a ClC-2 anion channel ortholog activated during meiotic maturation in C. elegans oocytes. Curr. Biol. 11, 161-170. Abstract Article

Samuel, A.D., Murthy, V.N., and Hengartner, M.O. (2001). Calcium dynamics during fertilization in C. elegans. BMC Dev. Biol. 1, 8. Abstract Article

Singson, A. (2001). Every sperm is sacred: fertilization in Caenorhabditis elegans. Dev. Biol. 230, 101-109. Abstract Article

Singson, A., Mercer, K.B., and L'Hernault, S.W. (1998). The C. elegans spe-9 gene encodes a sperm transmembrane protein that contains EGF-like repeats and is required for fertilization. Cell 93, 71-79. Abstract Article

Ward, S. and Carrel, J.S. (1979). Fertilization and sperm competition in the nematode Caenorhabditis elegans. Dev. Biol. 73, 304-321. Abstract 
Wilson, E.B. (1925). The Cell in Development and Heredity (New York: Macmillan).

Wissmann, A., Ingles, J., and Mains, P.E. (1999). The Caenorhabditis elegans mel-11 myosin phosphatase regulatory subunit affects tissue contraction in the somatic gonad and the embryonic epidermis and genetically interacts with the Rac signaling pathway. Dev. Biol. 209, 111-127. Abstract Article

Xu, X.Z., and Sternberg, P.W. (2003). A C. elegans sperm TRP protein required for sperm-egg interactions during fertilization. Cell 114, 285-297. Abstract Article

Yin, X., Gower, N.J., Baylis, H.A., and Strange, K. (2004). Inositol 1,4,5-trisphosphate signaling regulates rhythmic contractile activity of myoepithelial sheath cells in Caenorhabditis elegans. Mol. Biol. Cell 15, 3938-4399. Abstract Article

Zannoni, S., L'Hernault, S.W., and Singson, A.W. (2003). Dynamic localization of SPE-9 in sperm: a protein required for sperm-oocyte interactions in Caenorhabditis elegans. BMC Dev. Biol. 3, 10. Abstract Article

All WormBook content, except where otherwise noted, is licensed under a Creative Commons Attribution License 